\title{
Propuesta de un modelo de Corporate Foresight para pequeñas y medianas empresas ${ }^{1}$
}

\author{
Fernando Ortega San Martín \\ Universidad de Lima. Lima, Perú \\ Correo electrónico: fortegas@ulima.edu.pe
}

Recibido: 25/4/2012 / Aprobado: 17/5/2012

\begin{abstract}
Resumen: El Corporate Foresight es un enfoque relativamente nuevo de previsión del futuro orientado principalmente a las empresas, independientemente de su magnitud, para apoyar sus procesos de planeamiento de mediano y largo plazo. Si bien la compañía petrolera Royal Shell fue la primera empresa multinacional que empleó la prospectiva en sus procesos de planeamiento corporativo, su empleo por el sector privado no ha tenido la misma difusión e impacto que en el sector público. El presente artículo expone un modelo de Corporate Foresight para que pequeñas y medianas empresas puedan beneficiarse con la inteligencia anticipada que produce, y así mejorar su competitividad y sostenibilidad.
\end{abstract}

Palabras clave: Prospectiva empresarial / previsión / planificación estratégica / estudios de futuro / escenarios

\section{Proposal for a model of Corporate Foresight to SMEs}

Aвstract: Corporate Foresight is a relatively new approach to foresee the future, primarily oriented to business organizations, regardless of their size, in order to support their medium and long-term planning processes. Even though Royal Shell Oil Company was the first multinational company that used Foresight as planning tool, private sector worldwide has not used Foresight with the same success and impact that public sector. This article tries to introduce a Corporate Foresight model so that SMEs can be benefited from the anticipated intelligence it produces, and thus, improve their competitiveness and sustainability.

Keywords: Corporate Foresight / prospective / strategic planning / future studies / scenarios

1 Este trabajo forma parte de la investigación sobre Corporate Foresight que el autor viene desarrollando con el patrocinio del Instituto de Investigación Científica de la Universidad de Lima. 
Hay un pasado que se fue para siempre, pero hay un futuro que todavía es nuestro.

Frederick William Robertson (1816-1853).

\section{ANTECEDENTES}

Hay un punto en común en casi todas las culturas que han poblado la Tierra desde que la Humanidad creó la civilización tal como la entendemos: la preocupación por el futuro.

Por esta razón, según señala Medina (2000), Decoufle (1972, 1976) establece tres alternativas básicas de representación del futuro en la historia:

- el futuro como destino;

- el futuro como porvenir; y

- el futuro como devenir.

Desde el Extremo Oriente hasta las culturas americanas precolombinas, desde las tierras árticas hasta el sur de África, en todas ellas se desarrollaron diferentes prácticas para conocer el futuro: oráculos, astrología, cartomancia, i-ching, etcétera.

Sin embargo, más allá de las creencias religiosas imperantes en las distintas sociedades, donde se creía que el futuro como destino estaba en manos de Dios, los filósofos intentaron desarrollar un pensamiento sistemático y coherente sobre la formación del futuro. Eso se hizo evidente desde el inicio del Renacimiento, a partir del trabajo pionero de Tomás Moro y su obra Utopía (1516). ${ }^{2}$ Según Kateb (1977), el enfoque había cambiado hacia "el futuro como porvenir", tal como lo señala acertadamente Medina (2006).

La historia del pensamiento sobre el futuro no se detuvo. A las ideas revolucionarias de El contrato social de Jean-Jacques Rousseau (1762), le sucedió la Revolución Industrial (1768), que devino en un rápido desarrollo productivo y social, que generó gran expectativa en la sociedad por los impactos positivos que se vislumbraban derivados de los desarrollos científicos y tecnológicos. Todo ello fue insumo para que en la

2 Título original: "Dē optimō rē pūblicae statü dēque novā ìnsulā Ūtopiā" (Del estado ideal de una república en la nueva isla de Utopía). 
literatura se gestara un movimiento que llevó la creatividad al límite: la ciencia-ficción, que hasta hoy nos trae — aprovechando también el cine, la televisión e internet-imágenes de un futuro próximo.

Sin embargo, el siglo XX comenzó muy mal para el pensamiento sobre el futuro. Primero, la Humanidad vivió los horrores de la Primera Guerra Mundial. Después, llegaron los maravillosos años veinte como un grito desesperado de la sociedad por querer olvidar lo ocurrido en Europa la década anterior. Sin embargo, todo ello acabó con el crack de la Bolsa de Wall Street en 1929, que fue el germen de la desilusión, la falta de esperanza y los rencores que desembocaron en lo que después sería la Segunda Guerra Mundial. Es decir, la primera mitad del siglo $\mathrm{XX}$ fue un tiempo perdido para los estudios del futuro.

Pero de las cenizas de la Segunda Guerra Mundial se levantó un nuevo despertar, gracias al esfuerzo del Vaticano y el deseo de muchos intelectuales y políticos de no volver a cometer los errores de una guerra de carácter universal, que, debido al arsenal nuclear de ambos bandos, pondría en peligro la continuidad de la raza humana. Así, a principios de los años cincuenta, se reunieron en Roma los principales filósofos de Estados Unidos, Inglaterra, Francia e Italia, para tratar de analizar y construir un futuro sin guerras.

Del debate de este grupo, entre los años cincuenta y setenta nacieron los principales enfoques de los estudios de futuro: Forecasting (Estados Unidos), technology foresight (Inglaterra), prospectiva (Francia) y previsión humana y social (Italia).

La evolución del pensamiento sobre el futuro no quedó allí. Ya a finales de la década de 1990, los ingleses reconocieron que la tecnología por sí misma no podía seguir dirigiendo el futuro del mundo, porque las fuerzas sociales y los valores éticos comenzaban a limitar los desarrollos tecnológicos, especialmente en el campo de la biotecnología y la ingeniería genética. Fue así como nace el Foresight como un enfoque más holístico, "pretendiendo dar una idea de los sucesos probables a los cuales será preciso adaptarse, conduciendo a decisiones inmediatamente ejecutables" (Medina, 2006).

Pero, a excepción del forecasting norteamericano, ni la prospectiva ni el Foresight eran mayormente empleados por las empresas, salvo por las grandes corporaciones. Ya en 1968, la Royal Shell había sido la pionera en el uso de la construcción de escenarios como herramienta de 
planeamiento de largo plazos, habiendo identificado con varios años de antelación la crisis del petróleo de 1973 (Van der Heijden, 1998).

Justamente, para llevar el Foresight a las empresas, la consultora alemana Z_punkt GMBH, liderada por Cornelia Daheim, en el 2002 comenzó a sistematizar la experiencia de las empresas en todo el mundo en el uso de las diferentes herramientas de planeamiento de mediano y largo plazos. Así nace el Corporate Foresight, cuya difusión a lo largo de estos años ha sido importante, especialmente en Europa, Asia y Norteamérica. Esto ha facilitado la forma en que las empresas pueden construir su futuro, identificando y evaluando los principales factores que van a influir sobre su negocio en los próximos años (Daheim \& Uers, 2006).

No obstante, el éxito mundial del Corporate Foresight no ha llegado aún a América Latina. Esa es la razón por la cual, el objetivo de la investigación que se viene desarrollando en el Instituto de Investigación Científica de la Universidad de Lima es elaborar un manual de Corporate Foresight adaptado a la realidad de la empresa latinoamericana, que en gran parte son medianas y pequeñas (mypes), con limitados recursos para dedicarlos al análisis del futuro.

\section{DEFINICIÓN E IMPORTANCIA DEL CORPORATE FORESIGHT (CF)}

Corporate Foresight es un nuevo enfoque de planeamiento empresarial para adecuar las capacidades de las organizaciones a la creciente complejidad e indeterminación del mundo en que vivimos.

La importancia del uso del CF en las empresas puede resumirse principalmente en dos campos:

- Desarrollo de la inteligencia anticipatoria: para identificar los cambios en la conducta del consumidor y conocer la posible demanda futura, así como identificar las nuevas tecnologías que estarían vigentes en los próximos años (incluso aquellas desarrolladas por los competidores).

- Mejora de la formulación de estrategias y la definición de las prioridades: desarrollo de nuevos mercados y nuevos productos (innovaciones), inversión en infraestructura o en $\mathrm{I}+\mathrm{D}+\mathrm{i}$, decidir fusiones y adquisiciones o salidas programadas de mercados, etcétera. 


\section{CÓMO REALIZAR CORPORATE FORESIGHT EN UNA EMPRESA}

El proceso de establecimiento de acciones de Corporate Foresight en una empresa no resulta muy distinto a los procesos de la prospectiva clásica. Tal como lo establece Medina (2000), el ciclo permanente del pensamiento prospectivo consta de cuatro fases (figura 1):

a) La anticipación, actividad por la que los trabajos prospectivos son más conocidos y empleados, principalmente por generar información anticipada sobre hechos (rupturas), oportunidades y amenazas que podrían afectar la sostenibilidad futura de la empresa.

b) La apropiación de la información proporcionada por parte de la alta dirección de la empresa (accionistas, directores, gerentes), paso importante para transformarla en 'conocimiento anticipado'.

c) La acción, es decir, la toma de decisiones normativas, estratégicas y operativas basadas conscientemente en el conocimiento anticipado, dandole a este un valor estratégico para el futuro de la empresa, pasando a convertirse en 'inteligencia anticipada'.

d) El aprendizaje, que implica no solo la retroalimentación que nos proporcionará el propio futuro a medida que nos vaya llegando, sino también la forma como la empresa hace posible que el esfuerzo de $\mathrm{CF}$ se convierta en un proceso continuo y permanente, y no sea solamente "flor de un día".

Figura 1

El ciclo permanente del proceso prospectivo

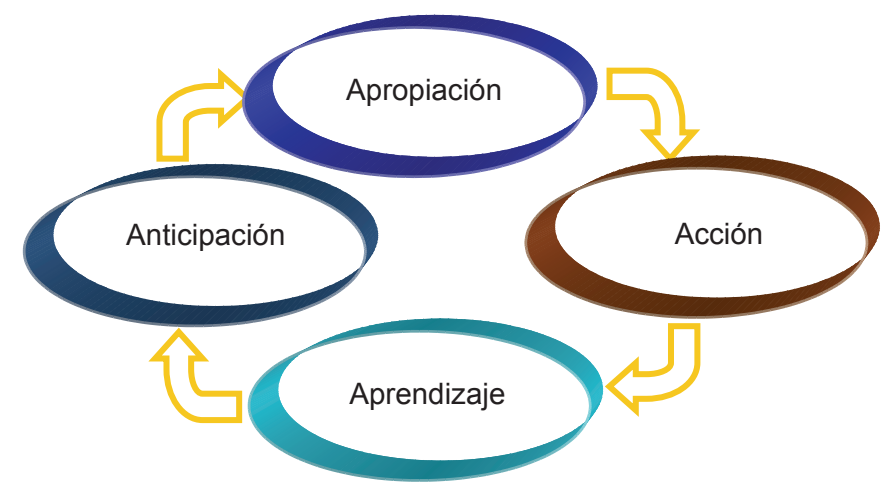

Fuente: Medina (2000). 
Dentro del marco expuesto en este artículo, donde también queremos respetar el código de buenas prácticas para estudios de Foresight planteados por Miles et al. (2008), proponemos que el desarrollo de actividades de $\mathrm{CF}$ en una empresa debe seguir el siguiente procedimiento:

Tabla 1

Propuesta de buenas prácticas para estudios de Foresight

\begin{tabular}{|c|c|c|c|}
\hline ETAPA & OBJETIVO & ACCIONES & RESPONSABLE \\
\hline 1 & $\begin{array}{l}\text { Generar una cultura } \\
\text { de pensamiento } \\
\text { prospectivo en la } \\
\text { empresa }\end{array}$ & $\begin{array}{l}\text { - Seleccionar a los actores internos } \\
\text { y externos que participarán en los } \\
\text { procesos de CF en la empresa. } \\
\text { - Desarrollar charlas motivacionales } \\
\text { con los actores seleccionados. } \\
\text { - De ser posible, hacer participar a } \\
\text { los ejecutivos de la empresa en } \\
\text { procesos de Foresight que se eje- } \\
\text { cuten paralelamente en el mundo } \\
\text { sobre temas vinculados a la activi- } \\
\text { dad de la empresa. }\end{array}$ & $\begin{array}{l}\text { Alta dirección, con } \\
\text { el apoyo de un con- } \\
\text { sultor externo de } \\
\text { ser necesario. }\end{array}$ \\
\hline 2 & $\begin{array}{l}\text { Seleccionar a los } \\
\text { responsables de las } \\
\text { actividades de CF } \\
\text { en la empresa (Task } \\
\text { Force) }\end{array}$ & $\begin{array}{l}\text { - Evaluar el potencial creativo y la } \\
\text { orientación hacia el futuro (actitud } \\
\text { proactiva) del personal ejecutivo. } \\
\text { - Seleccionar un equipo multidisci- } \\
\text { plinario y multiárea (producción, } \\
\text { márketing, finanzas, I+D, pos- } \\
\text { venta, etcétera) con la finalidad de } \\
\text { mantener un enfoque holístico en } \\
\text { el proceso de CF. } \\
\text { - Capacitar en técnicas prospecti- } \\
\text { vas al equipo seleccionado. }\end{array}$ & $\begin{array}{l}\text { Alta dirección, con } \\
\text { el apoyo de un con- } \\
\text { sultor externo. }\end{array}$ \\
\hline 3 & $\begin{array}{l}\text { Establecer los focos } \\
\text { de atención de los } \\
\text { estudios específicos } \\
\text { de CF y los horizon- } \\
\text { tes temporales }\end{array}$ & $\begin{array}{l}\text { Desde el principio resulta conve- } \\
\text { niente identificar los temas espe- } \\
\text { cíficos y los objetivos hacia donde } \\
\text { apuntar los esfuerzos de CF. No } \\
\text { debe limitarse solo a los mercados } \\
\text { actuales, sino que debe incluirse a } \\
\text { los mercados potenciales. } \\
\text { - Los horizontes temporales deben } \\
\text { ser señalados explícitamente. Se } \\
\text { recomienda que sean como míni- } \\
\text { mo de tres años en adelante, y no } \\
\text { más allá de diez años. }\end{array}$ & $\begin{array}{l}\text { Alta dirección y } \\
\text { Task Force (grupo } \\
\text { responsable) }\end{array}$ \\
\hline
\end{tabular}

(continúa)

3 Si bien la prospectiva puede trabajar con horizontes de treinta años, en el caso del CF se recomienda no exceder los diez años, porque de esa manera los resultados pueden ser más creíbles por los miembros de la organización. 
(continuación)

\begin{tabular}{|c|c|c|c|}
\hline ETAPA & OBJETIVO & ACCIONES & RESPONSABLE \\
\hline 4 & $\begin{array}{l}\text { Planeamiento de los } \\
\text { esfuerzos de CF }\end{array}$ & $\begin{array}{l}\text { El Task Force debe definir clara- } \\
\text { mente: metodologías a emplear } \\
\text { (selección de herramientas pros- } \\
\text { pectivas), fuentes de información, } \\
\text { presupuesto y cronograma de ac- } \\
\text { tividades. } \\
\text { - Resulta recomendable que tam- } \\
\text { bién el Task Force formule una } \\
\text { propuesta de modelo a seguir por } \\
\text { la empresa al finalizar los primeros } \\
\text { trabajos de CF para darle continui- } \\
\text { dad al proceso. }\end{array}$ & Task Force \\
\hline 5 & $\begin{array}{l}\text { Desarrollo del pri- } \\
\text { mer proceso de CF } \\
\text { en la empresa }\end{array}$ & $\begin{array}{l}\text { - Aplicación de las herramientas } \\
\text { metodológicas. } \\
\text { - Validación de cada parte del pro- } \\
\text { ceso. } \\
\text { - Identificación de los drivers cla- } \\
\text { ves para la empresa, así como las } \\
\text { posibles rupturas, oportunidades y } \\
\text { amenazas. } \\
\text { - Identificación de los escenarios fu- } \\
\text { turos para la empresa, y selección } \\
\text { del escenario-meta. } \\
\text { - Propuesta de construcción del } \\
\text { escenario-meta. }\end{array}$ & Task Force \\
\hline 6 & $\begin{array}{l}\text { Empleo efectivo de } \\
\text { la información de } \\
\text { inteligencia anticipa- } \\
\text { toria generada por } \\
\text { el esfuerzo desarro- } \\
\text { llado en CF }\end{array}$ & $\begin{array}{l}\text { - Análisis de la información (apro- } \\
\text { piación interna de los resultados). } \\
\text { - Toma de decisiones a nivel norma- } \\
\text { tivo, estratégico y operativo. }\end{array}$ & $\begin{array}{l}\text { Accionistas, alta di- } \\
\text { rección y ejecutivos }\end{array}$ \\
\hline 7 & $\begin{array}{l}\text { Sostenibilidad del } \\
\text { proceso de CF en la } \\
\text { empresa }\end{array}$ & $\begin{array}{l}\text { - Definición de la estructura perma- } \\
\text { nente de CF. } \\
\text { - Designación de los responsables } \\
\text { permanentes de CF dentro de la } \\
\text { empresa. } \\
\text { - Seguimiento permanente de los } \\
\text { drivers claves e identificación de } \\
\text { drivers emergentes. } \\
\text { - Elaboración periódica de informa- } \\
\text { ción de CF para la alta dirección. }\end{array}$ & $\begin{array}{l}\text { Alta dirección } \\
\text { y responsables } \\
\text { permanentes de CF }\end{array}$ \\
\hline
\end{tabular}

Fuente: Miles et al. (2008).

4 La validación se puede realizar solo con miembros de la organización, aunque se recomienda la participación de personas externas (expertos, proveedores, clientes, etcétera). 
Las etapas anteriormente mostradas generan un modelo de aplicación de Corporate Foresight en una organización. Hay varios modelos vigentes, propuestos, por ejemplo, por Peter (2006), Daheim y Uerz (2006) y últimamente por Rohrbeck (2011), pero ellos están principalmente orientados a grandes corporaciones, que suelen disponer de importantes recursos para realizar estudios de futuro específicos para tales organizaciones: unidades especializadas en prospectiva dentro de sus organigramas o presupuestos asignados para la contratación de servicios de consultoría en prospectiva y estudios del futuro. En la figura 2 se muestra en el modelo propuesto por Peter (2006).

Figura 2

\section{Prospectiva y estudios de futuro}

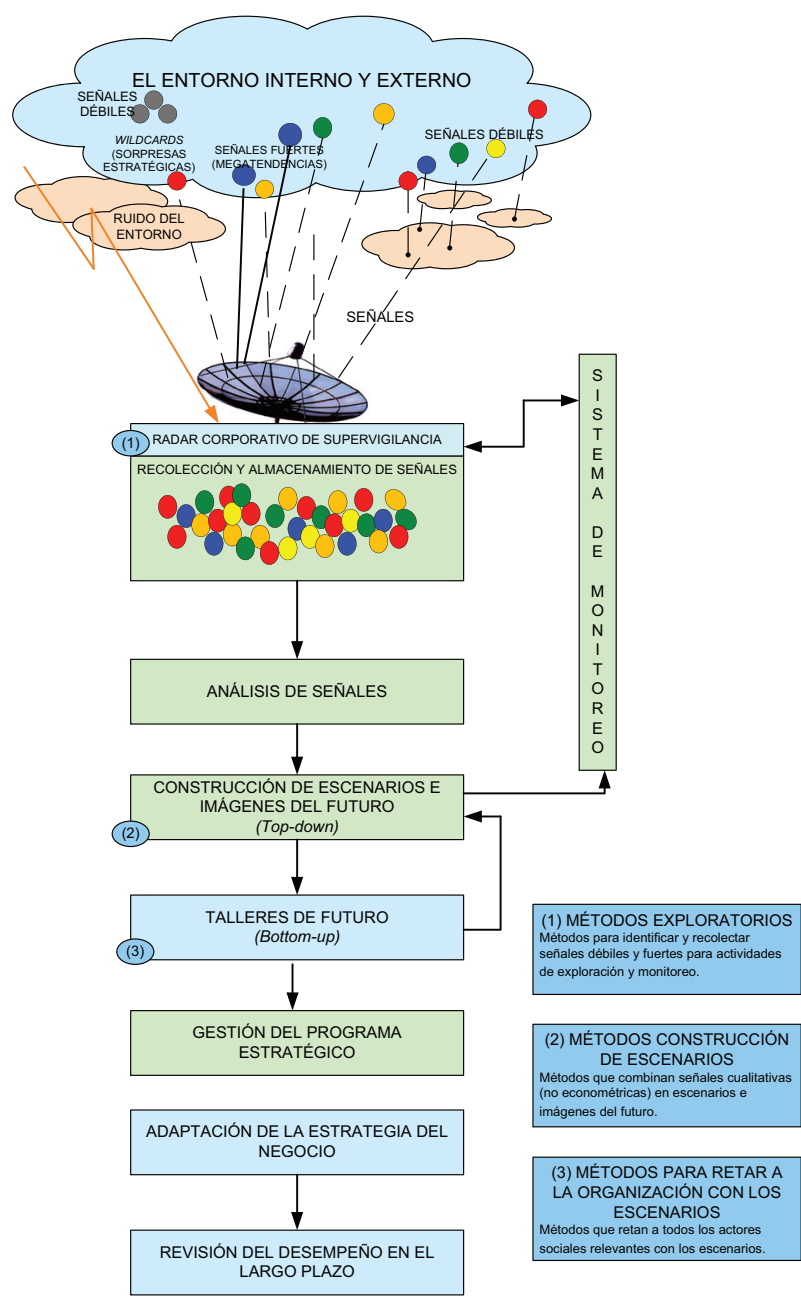


Cuhls y Johnston (2006) y principalmente Jeong, Park y Heo (2007) han realizado un análisis de estos modelos, que se muestran en la tabla siguiente:

Tabla 2

Tipología de modelos de organización de Corporate Foresight

\begin{tabular}{|c|c|c|c|}
\hline $\begin{array}{l}\text { Tipo de organización } \\
\text { de Corporate } \\
\text { Foresight }\end{array}$ & Características & Función principal & Ejemplos \\
\hline $\begin{array}{l}\text { El puesto de recolec- } \\
\text { ción de información }\end{array}$ & $\begin{array}{l}\text { - Bajo grado comparativo de } \\
\text { actividades de Foresight } \\
\text { - Los estudios se realizan } \\
\text { mayormente en conjunto con } \\
\text { otras actividades de I+D, y } \\
\text { fuertemente integrados a } \\
\text { ellas). } \\
\text { - Las personas responsables } \\
\text { del Foresight son solo "futuris- } \\
\text { tas" de tiempo parcial. } \\
\text { - Rara vez forman una unidad } \\
\text { separada. }\end{array}$ & $\begin{array}{l}\text { - Proveen información bási- } \\
\text { ca del entorno para proce- } \\
\text { sos de toma de decisiones } \\
\text { en estas áreas. } \\
\text { - Se enfocan en la búsqueda } \\
\text { y colecta de información } \\
\text { relativa al futuro que ya ha } \\
\text { sido preparada por otros. }\end{array}$ & $\begin{array}{l}\text { Procter \& Gamble } \\
\text { Volvo } \\
\text { Lufthansa }\end{array}$ \\
\hline El observatorio & $\begin{array}{l}\text { - Una unidad autónoma de } \\
\text { Foresight con un staff a tiem- } \\
\text { po completo y con presupues- } \\
\text { to propio. } \\
\text { - Redes internas y fuentes ex- } \\
\text { ternas de información. } \\
\text { - Pero las redes son mayor- } \\
\text { mente compuestas por espe- } \\
\text { cialistas de campos similares, } \\
\text { y solo rara vez cubren áreas } \\
\text { más amplias de Foresight. }\end{array}$ & $\begin{array}{l}\text { - Se enfocan en un altamen- } \\
\text { te especializado y muy sin- } \\
\text { gular campo de utilidad de } \\
\text { la empresa. } \\
\text { - Reúsan datos ya existen- } \\
\text { tes y, regularmente, tam- } \\
\text { bién generan nuevo cono- } \\
\text { cimiento relacionado con el } \\
\text { futuro. }\end{array}$ & $\begin{array}{l}\text { IBM } \\
\text { DB } \\
\text { Eni }\end{array}$ \\
\hline El think tank & $\begin{array}{l}\text { - Realizan el trabajo más am- } \\
\text { plio y elaborado de Foresight. } \\
\text { - Forman un grupo selecto de } \\
\text { futuristas de tiempo comple- } \\
\text { to, expertos e investigadores. } \\
\text { - Rango mucho más amplio de } \\
\text { tareas que un observatorio. } \\
\text { - Construyen una red global de } \\
\text { expertos tanto de dentro de } \\
\text { la empresa como de fuera. }\end{array}$ & $\begin{array}{l}\text { - Explora toda clase de te- } \\
\text { mas relacionados con el } \\
\text { futuro no solo en el entorno } \\
\text { inmediato de la empresa } \\
\text { sino también en la más am- } \\
\text { plia esfera socioeconómi- } \\
\text { ca, cultural y regional. }\end{array}$ & $\begin{array}{l}\text { BT } \\
\text { Ericsson } \\
\text { Daimler-Chrysler } \\
\text { Philips } \\
\text { Siemens }\end{array}$ \\
\hline
\end{tabular}

Fuente: Jeong, Park y Heo (2007). 
Incluso, sobre este tema en particular, Daheim y Uerz (2008) añaden una cuarta tipología a la que llaman el outsourcer, que se caracteriza por ser:

[...] una unidad de Corporate Foresight que identifica y define el tema y metas de un proceso de CF desde su perspectiva de experto dentro de la empresa, pero a menudo permite que el proceso mismo o grandes partes de la investigación involucrada sea conducida por una organización externa (consultora) antes de reintegrar y utilizar los resultados en el planeamiento estratégico u otros campos al interior de la empresa. El proceso dentro de la empresa es a menudo conducido por un equipo de proyecto seleccionado en relación a las tareas y temas específicos en los que se concentrará el proceso de Foresight, y aunque la unidad es más bien de pequeño tamaño, tiene una alta visibilidad dentro y fuera de la empresa. La unidad tendrá un alto nivel de experticia en CF, pero la mayoría del trabajo del día a día (como el monitoreo de tendencias) es simplemente contratado a terceros.

Como puede observarse, ninguno de esos modelos puede ser aplicable a una pequeña o mediana empresa latinoamericana, ${ }^{5}$ debido a que según Álvarez y Durán (2009):

- dispone de recursos financieros limitados, que son priorizados para el cumplimiento de metas operativas (de corto plazo);

- carece de recursos humanos altamente especializados, principalmente en materia de planeamiento; $y$

- dispone de fuentes de información limitadas, generalmente ligadas directamente a las actividades propias de la empresa, lo que sesga cualquier análisis e impide concebir y utilizar enfoques holísticos.

Conociendo esta realidad de la pequeña y mediana empresa latinoamericana, en la figura 3 se aprecia el modelo que proponemos en este artículo, que está orientado principalmente a fomentar el uso del Corporate Foresight en estas empresas, que, al igual que las grandes corporaciones, también necesitan realizar estudios de futuro para asegurar su sostenibilidad.

5 Para efectos de esta investigación se ha tomado la definición del Banco Mundial para pequeñas empresas (entre 10 y 50 trabajadores y activos hasta 3 millones de dólares) y medianas empresas (entre 51 y 300 trabajadores y activos hasta US\$ 15 millones). 
Nótese que, a pesar de su aparente simplicidad en comparación con los modelos para grandes corporaciones, los procesos considerados en la figura 3 permiten asegurar un nivel de análisis adecuado, capaz de generar un set de escenarios posibles de significativa utilidad para la definición de estrategias.

Figura 3

Modelo de aplicación del Corporate Foresight para pymes

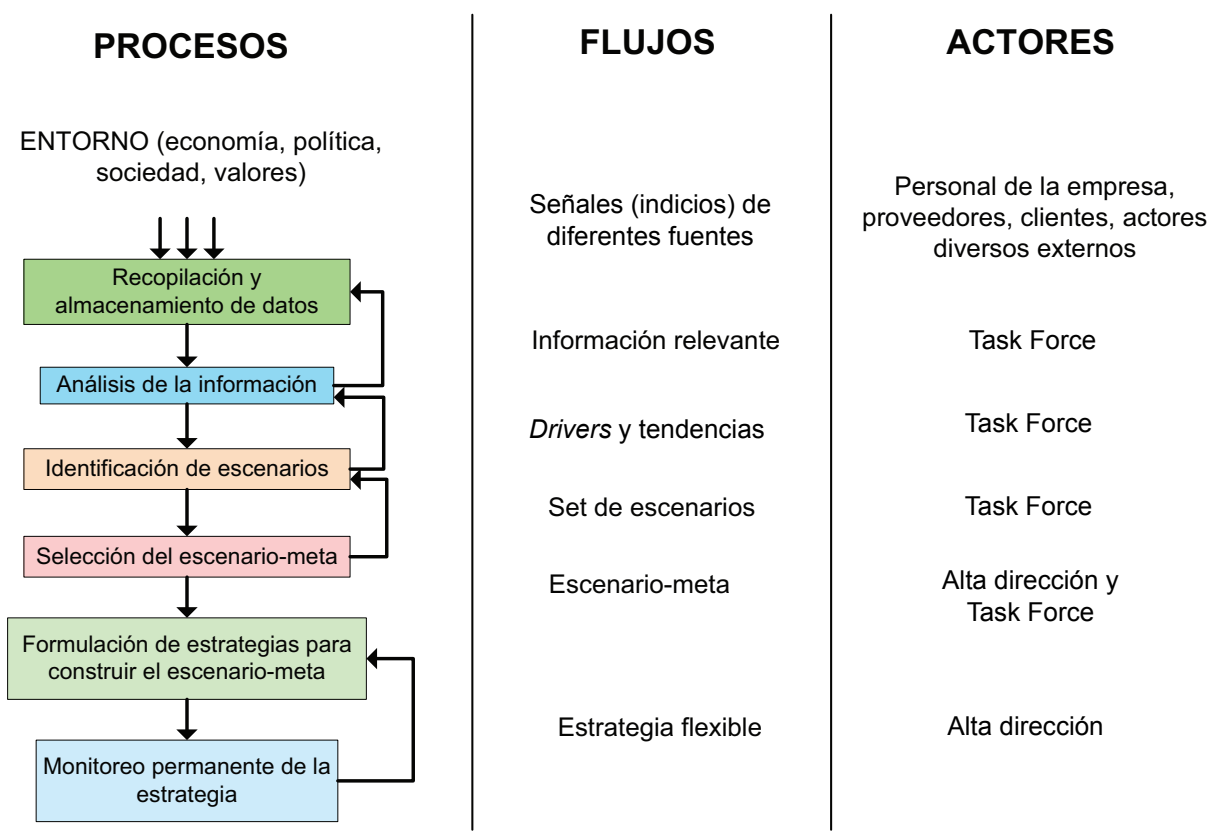

Elaboración propia.

Pasemos a analizar cada uno de los procesos mostrados en la figura 3, pero antes, analicemos el entorno de donde vamos a extraer la información. Existe una natural propensión en las pymes a enfocar la búsqueda de información solo en lo más cercano a la organización: el entorno próximo (proveedores, clientes, competidores). Pero eso no es suficiente y menos ahora que vivimos en una sociedad cada vez más compleja e indeterminada. Hay que extender la búsqueda de información hacia el entorno más lejano, pues el futuro se va a ir formando en esos espacios, donde confluyen, entre otros, la regulación, la aparición de nuevas tecnologías, la introducción de productos sustitutos, los cambios en los gustos y preferencias de los consumidores, incluso los 
cambios en los valores de la sociedad. Eso necesariamente implica un esfuerzo mayor por parte de la organización, pero el trabajo extra bien lo vale.

Tal como lo hemos señalado, una de las decisiones claves que tiene que tomar la alta dirección de la empresa es la selección del 'Task Force' (grupo de trabajo). Se espera que sus integrantes sean miembros jóvenes de la organización, aunque con algunos años de experiencia en el sector y en la empresa. Por lo menos el líder del grupo debe conocer técnicas prospectivas, aunque no se debe descartar que un consultor externo especializado en prospectiva acompañe todo el proceso, para darle consistencia a todo lo actuado.

La recopilación de la información sobre el futuro debe responder a un plan sistemático que cubra todas las fuentes relevantes disponibles: bases de datos internas y externas, diarios y revistas especializados en temas políticos, económicos, comerciales, tecnológicos y sociales, páginas web y blogs en internet, patentes, estudios de prospectiva previamente elaborados, etcétera. Los miembros del Task Force deben reconocer incluso las señales débiles que genera la tecnología, la economía y la sociedad, y que pueden tener potencial de convertirse en "hechos portadores de futuro", eventos que pueden causar un gran impacto en el futuro del tema bajo estudio, pero que en el presente poseen una muy baja probabilidad de ocurrencia. Un "hecho portador del futuro" puede ser crucial para nuestra actividad. Por ejemplo, si estuviéramos analizando el futuro de una empresa del campo del entretenimiento, ¿se pueden imaginar los cambios sociales, económicos y de valores y actitudes que generaría el descubrimiento y puesta al mercado de una vacuna contra el VIH-sida?

Se recomienda que la información sea almacenada en una base de datos interna de la organización, clasificada fundamentalmente en tres categorías: drivers, tendencias y hechos portadores de futuro. Los drivers o vectores de futuro son variables o fenómenos cuyos movimientos en magnitud y sentido pueden determinar cambios notorios en el futuro. Las tendencias son fuerzas sociales internamente consistentes que mueven a la Humanidad en un sentido determinado en un período dado. Entre ambos (drivers y tendencias) existe una profunda relación: toda tendencia está formada por drivers. 
Generalmente no resulta sencillo identificar directamente a los drivers y las tendencias. Por ello, se suelen emplear una serie de herramientas metodológicas denominadas "métodos exploratorios": ${ }^{6}$ Paneles de Expertos, Environmental Scanning, Análisis de Tendencias, Análisis Morfológico, Relevance Tree, entre otros.

Pero no basta con identificar drivers, tendencias y hechos portadores de futuro. Es preciso validarlos, porque resulta fácil creer en lo que hemos trabajado, aunque esté errado. Necesitamos confrontar lo avanzado por el Task Force, con la opinión de expertos. Para realizar esta tarea se dispone de métodos de validación, de los cuales los más empleados son dos: la Encuesta Delphi y el Ábaco de Regnier.

Ambas herramientas no solo sirven para validar los drivers que nos permiten saber si estamos en el buen camino o si nos hemos desviado hacia temas no relevantes, sino que sirven sobre todo para calificar a cada driver en dos dimensiones claves: importancia e incertidumbre, insumos valiosísimos para el ulterior método de construcción de escenarios, que es el punto de mayor expectativa del estudio.

Un escenario es, ante todo, una visión consistente de futuro, que se construye a partir de la combinación de los drivers validados, y que tradicionalmente toma la forma de una narración, aunque cada día se emplean más las técnicas audiovisuales, dada su amplia difusión y costos cada vez menores.

Como suele ocurrir con los métodos más difundidos y empleados, se han generado diversas maneras o estilos de construcción de escenarios. Quizás la variante más usada es la de los "Ejes de Schwartz", llamada así por haber sido desarrollada por Peter Schwartz (1991), uno de los futuristas más importantes de Estados Unidos. La práctica de este método es muy sencilla y permite agrupar los drivers en cuatro cuadrantes producto de la intersección de los dos ejes principales: importancia e incertidumbre (figura 4).

6 En este artículo no se explicarán en detalle las diferentes metodologías empleadas en los estudios de Foresight. Recomendamos la revisión de "Future Research Methodology v.3.0", CD elaborado por el Millennium Project, que presenta las 38 herramientas metodológicas más empleadas en estudios de prospectiva. 


\section{Figura 4}

Los cuatro cuadrantes generados por los Ejes de Schwartz

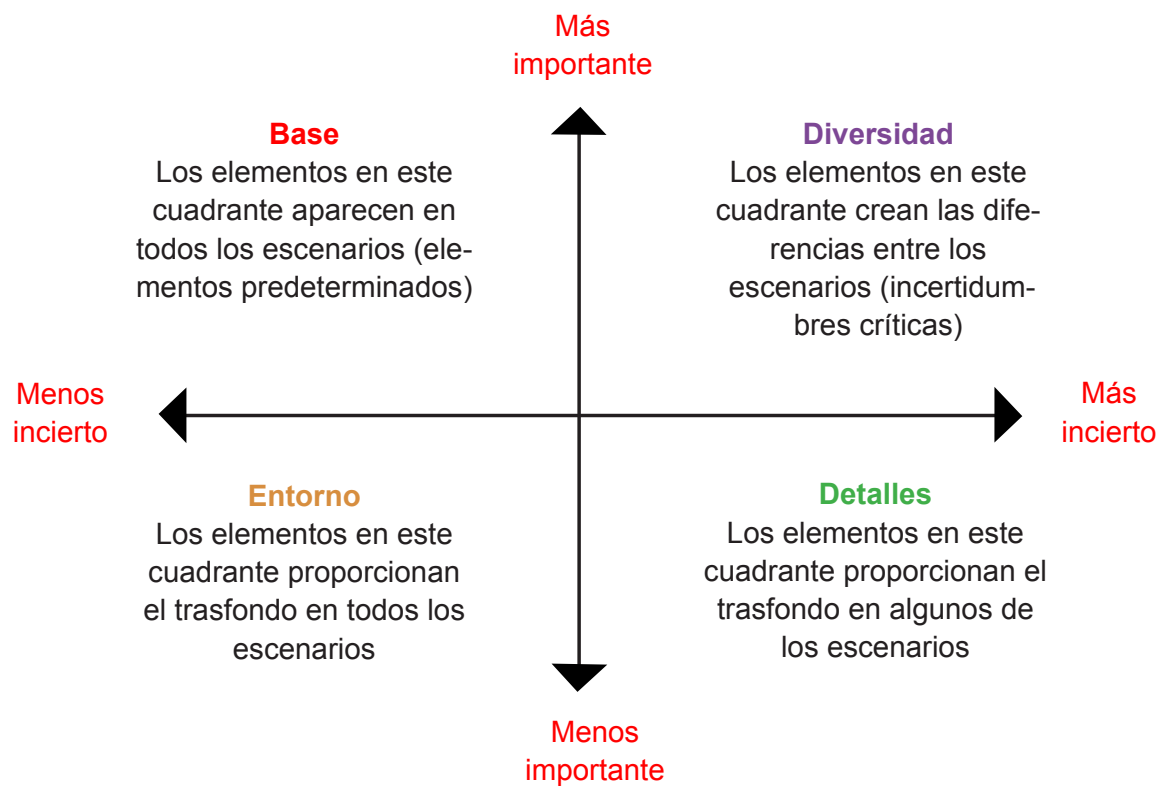

Fuente: Johnston (2002).

Los escenarios diferenciados nacen de la combinación de los drivers del cuadrante de la Diversidad, aunque es preciso señalar que todos los escenarios comparten el mismo Entorno y la misma Base; entonces podríamos decir que escenario (i) = Entorno + Base + Diversidad (i).

No es posible definir a priori el número de escenarios que pueden presentarse, aunque siempre se trata de que sea un número relativamente pequeño, no más de ocho, porque es una cantidad más manejable para la empresa. Hay que tener presente que el objetivo no es identificar los escenarios, trabajo de por sí complejo, sino, sobre todo, diseñar las estrategias para cada uno de los escenarios identificados. Eso es lo ideal, aunque por razones prácticas, de costo y de tiempo, lo que generalmente se hace es formular una sola estrategia, la referida al escenario-meta (aquel que deseamos construir), pero reforzándola ante la eventualidad de que aparezcan los escenarios menos deseables.

En la formulación de estrategias se recomienda emplear el método de Backcasting para establecer los hitos temporales que la organización debe alcanzar en su ruta hacia la construcción del escenario-meta. 
El no cumplimiento de un hito temporal alertará a la organización que no se están desarrollando los planes como estaban previstos y permitirá tomar las acciones respectivas.

Por lo expuesto, el set de herramientas metodológicas que recomendamos emplear sería el siguiente:

Tabla 3

Herramientas metodológicas sugeridas para el Corporate Foresight en pymes

\begin{tabular}{|l|l|}
\hline Etapa del proceso & Herramientas recomendadas \\
\hline Identificación de drivers & $\begin{array}{l}\text { - Environmental Scanning } \\
\text { - Análisis de tendencias } \\
\text { - Análisis morfológico } \\
\text { - Relevance Tree }\end{array}$ \\
\hline Validación de drivers & $\begin{array}{l}\text { - Encuesta Delphi } \\
\text { - Ábaco de Regnier }\end{array}$ \\
\hline Identificación de escenarios & $\begin{array}{l}\text { - Construcción de escenarios (Ejes de } \\
\text { Schwartz) }\end{array}$ \\
\hline Formulación de estrategias & Backcasting \\
\hline
\end{tabular}

Elaboración propia.

Pero no olvidemos lo mostrado en la figura 1. El proceso prospectivo es permanente, y debemos tener siempre presente la correspondiente retroalimentación, producto de contrastar el escenario-meta con la realidad. Recordemos que la prospectiva no es predictiva, y por lo tanto, siempre hay una variedad de posibilidades de escenarios que pueden presentarse. De ahí que sea preciso el monitoreo continuo y permanente de los principales drivers y tendencias que han permitido la construcción del escenario-meta. Ante cualquier variación significativa de los movimientos de los drivers y ante un comportamiento de mayor o menor intensidad de una tendencia, se recomienda volver a construir los escenarios, para comprobar si no hay un nuevo escenario-meta más conveniente para la empresa.

\section{CONCLUSIONES}

Ante la creciente complejidad e indeterminación del mundo, ya no es posible mantener los sistemas tradicionales de planeamiento empresa- 
rial. El enfoque del Corporate Foresight resulta entonces una alternativa viable y saludable para aquellas organizaciones preocupadas por establecer sistemas internos de alerta temprana de las oportunidades y amenazas que podrían impactar su futuro.

Gracias a que el Corporate Foresight emplea herramientas metodológicas relativamente sencillas, se espera que su uso por parte de pequeñas y medianas empresas sea ampliamente difundido.

\section{REFERENCIAS}

1. Alsan, A., \& Oner, M. A. (2003). An integrated view of Foresight: integrated Foresight management model, Foresight 5(2), 33-45.

2. Álvarez, M., \& Durán, J. (2009), Manual de la micro, pequeña y mediana empresa: una contribución a la mejora de los sistemas de información y el desarrollo de las políticas públicas. San Salvador: GTZ Gmbh y Cepal.

3. Ayyagari, M., Beck, T., \& Demirguc-Kunt, A. (2003). Small and medium enterprises across the globe: a new database. Policy Research Working Paper Series. Washington D.C.: The World Bank.

4. Becker, P. (2002). Corporate Foresight in Europe: a first overview. Bruselas: Cordis Working Paper.

5. Burmeister, K., \& Neef, A. (2005). In the long run. Munich: Z-punkt.

6. Burmeister, K. (2009). Corporate Foresight - a 360-Degree radar on business rooftops. Munich: Z-punkt.

7. Burmeister, K., Neef, A. S., \& Beyers, B. (2005). Corporate Foresight. Munich: Z-punkt.

8. Cuhls, K., \& Johnston, R. (2006). Corporate FTA (Future-oriented technology analysis). Paper presentado en el Seminario Internacional sobre Future-Oriented Technology Analysis: Impact of FTA Approaches on Policy and Decision-Making, organizado por el IPTS, Sevilla, 28-29 de setiembre del 2006.

9. Daheim, C., \& Uerz, G. (2006). Corporate Foresight in Europe: ready for the next step. Paper presentado en el Segundo Seminario Internacional sobre Future-Oriented Technology Analysis: Impact of FTA Approaches on Policy and Decision-Making, organizado por el IPTS, Sevilla, 28-29 de setiembre del 2006. 
10. Daheim, C., \& Uerz, G. (2008). Corporate Foresight in Europe: from trend based logics to open Foresight. Technology Analysis \& Strategic Management, 20(3), 321-336.

11. Decoufle, A. C. (1972). La prospective. Que-sais-je? París: Presses Universitaires de France.

12. Decoufle, A. C. (1976). Sociologie de la prevision: l'exemple de la prospective sociale en France. París: Presses Universitaires de France.

13. Georghiou, L., Cassingena-Harper, J., Keenan, M. \& Popper, R. (2008). The Handbook of Technology Foresight. Cheltenham: Edward Elgar Publishing Limited.

14. Glenn, J. C., Gordon, T. J., \& Florescu, E. (2011). The state of the future 2011. Washington D.C.: The Millennium Project.

15. Glenn, J. C., \& Gordon, T. (2010). Futures research methodology version 3.0. Washington D.C.: The Millennium Project.

16. Jannek, K. \& Burmeister, K. (2007). Corporate Foresight in small and medium-sized enterprises. The European Foresight Monitoring Network: Foresight Brief, 101, pp. 1-4.

17. Jeong, N.; Park, J. \& Heo, T. (2007). Conceptual designs for trend analysis system: a tool for Corporate Foresight. Gangwon-Do. Ponencia presentada en la $9^{\text {a }}$ Conferencia Internacional sobre Tecnologías de Comunicación Avanzadas - ICACT, organizada por el Ministerio de Información y Comunicación. Phoenix Park (Corea del Sur), 12-14 de febrero del 2007.

18. Kateb, G. (1977). Utopismo. Enciclopedia Internacional de las Ciencias Sociales, 10, 597-600.

19. Medina, J. (2000). "La construcción social del futuro. Anotaciones desde la previsión humana y social". Ponencia presentada en el IV Encuentro Iberoamericano de Estudios Prospectivos, organizado por la Universidad de La Habana, 31 de enero del 2000.

20. Medina, J. (2006). Map of levels of complexity and indetermination for Foresight studies. Sevilla: IPTS.

21. Medina, J. \& Ortegón, E. (2006). Manual de prospectiva y decisión estratégica: bases teóricas e instrumentos para América Latina y el Caribe. Santiago de Chile: Ilpes-Cepal. 
22. Mietzner, D. \& Reger, G. (2005). Advantages and disadvantages of scenario approaches for strategic Foresight, International Journal of Technology Intelligence and Planning, vol. 1(2), pp. 220-239.

23. Miles, I., Georghiou, L., Cassingena Harper, J., Keenan, M., \& Popper, R. (2008). The handbook of technology Foresight. Cheltenham: Edward Elgar Publishing Limited.

24. Moura, P. (1994). Construindo o futuro. O impacto global do novo paradigma. Río de Janeiro: Mauad Editorial.

25. Organización de las Naciones Unidas para el Desarrollo Industrial (ONUDI) (2005). Technology Foresight Manual. Viena: ONUDI.

26. Peter, M. K. (2006). Future screening: A best practice framework for advanced strategic Corporate Foresight. Recuperado el 16 de abril de 2011, de http://futurescreening.com/foresightframework.htm

27. Ringland, G. (1998). Scenario Planning: managing for the future. Chichester: John Wiley \& Sons Ltd.

28. Rohrbeck, R. (2011). Corporate Foresight, towards a maturity model for the future orientation of a firm. Berlín: Springer-Verlag.

29. Schwartz, P. (1991). The art of the long view: Paths to strategic insight for yourself and your company. Nueva York: Bantam.

30. Shell International Limited. (2005). Shell global scenarios to 2025. Londres: Royal Dutch/Shell Group.

31. Tan Hong Ngoh, E. \& Hoo Tiang Boon (2008). Thinking about the future-strategic anticipation and RAHS. Ponencia presentada en el Second International Risk Assessment and Horizon Scanning Symposium, organizado por el Centre of Excellence for National Security, Singapur, 12-14 de octubre del 2008.

32. Van der Heijden, K. (1998). Escenarios: El arte de prevenir el futuro. México D.F.: Panorama Editorial. 